\title{
Thin Film ZnO as Sublayer for Electric Contact for Bulk GaN with Low Electron Concentration
}

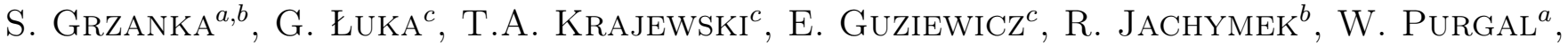 \\ R. Wiśniewska ${ }^{b}$, A. SArzyńska ${ }^{b}$, A. Bering-Staniszewska ${ }^{b}$, M. Godlewski $^{c, d}$ \\ AND P. PERLIN ${ }^{a, b}$ \\ ${ }^{a}$ TopGaN Ltd., Sokołowska 29/37, 01-142 Warsaw, Poland \\ ${ }^{b}$ Institute of High Pressure Physics, Sokołowska 29/37, 01-142 Warsaw, Poland \\ ${ }^{c}$ Institute of Physics, Polish Academy of Sciences, al. Lotników 32/46, 02-668 Warsaw, Poland \\ ${ }^{d}$ Department of Mathematics and Natural Sciences, College of Science, Cardinal Stefan Wyszyński University \\ Dewajtis 5, 01-815 Warsaw, Poland
}

\begin{abstract}
Fabrication of low resistivity ohmic contacts to $\mathrm{N}$ polarity gallium nitride crystal is an important issue for the construction of the vertical current flow devices like laser diodes and high brightness light emitting diodes. Gallium nitride is a challenging material because of the high metal work function required to form a barrier-free metal-semiconductor interface. In practice, all useful ohmic contacts to GaN are based on the tunneling effect. Efficient tunneling requires high doping of the material. The most challenging task is to fabricate high quality metal ohmic contacts on the substrate because the doping control is here much more difficult that in the case of epitaxial layers. In the present work we propose a method for fabricating low resistivity ohmic contacts on $\mathrm{N}$-side of GaN wafers grown by hydride vapor phase epitaxy. These crystals were characterized by a $n$-type conductivity and the electron concentration of the order of $10^{17} \mathrm{~cm}^{-3}$. The standard $\mathrm{Ti} / \mathrm{Au}$ contact turned out to be unsatisfactory with respect to its linearity and resistance. Instead we decided to deposit high- $n$ type $\mathrm{ZnO}$ layers (thickness $50 \mathrm{~nm}$ and $100 \mathrm{~nm}$ ) prepared by atomic layer deposition at temperature of $200{ }^{\circ} \mathrm{C}$. The layers were highly $n$-type conductive with the electron concentration in the order of $10^{20} \mathrm{~cm}^{-3}$. Afterwards, the metal contact to $\mathrm{ZnO}$ was formed by depositing $\mathrm{Ti}$ and $\mathrm{Au}$. The electrical characterization of such a contact showed very good linearity and as low resistance as $1.6 \times 10^{-3} \Omega \mathrm{cm}^{2}$. The results indicate advantageous properties of contacts formed by the combination of the atomic layer deposition and hydride vapor phase epitaxy technology.
\end{abstract}

PACS: 72.80.Ey, 73.40.Kp, 73.40.Lq

\section{Introduction}

It often comes as a surprise that in case of optoelectronic devices the fabrication of the ohmic contacts to the substrate material is more challenging than to the epitaxial layer. This is because we can, in large extent, control the doping of the epi-layer while the donor concentration in the bulk crystal is related to the growth method and cannot be easily modified. GaN crystals, available on the market, and fabricated usually by a hydride vapour phase epitaxy (HVPE) method suffers usually from the doping problems limiting the free electron concentration in the bulk of the material to $10^{17}-10^{18} \mathrm{~cm}^{-3}$. The fabrication of the low resistance ohmic contact to such material is a difficult task in case of GaN because the work function of the all available metals is too low to produce the ideal Schottky barrier-free metal-semiconductor interfaces. For modestly doped GaN, additionally, it is difficult to create a tunneling junction between metal and semiconductor.
It is quite natural to think about non-metallic electrodes whose electronic structure would fit better to this of $\mathrm{GaN}$. $\mathrm{ZnO}$ is an example of the semiconductor which can be easily highly $n$-type doped and having an energy gap almost identical to this of GaN. Additionally, atomic layer deposition (ALD) method provides an efficient way of low temperature growth of highly doped $n$-type $\mathrm{ZnO}$ layers [1]. It is also important that this method is compatible with the classical processing schemes of the nitride devices.

This paper presents the results of using thin $\mathrm{ZnO}$ layers as sub-metal electrodes layers in the processing of GaN devices. In the present work we demonstrate a method for fabricating low resistivity ohmic contacts on nitrogen side of GaN wafers grown by HVPE. These crystals were characterized by an $n$-type conductivity and the electron concentration in the order of $10^{17} \mathrm{~cm}^{-3}$. We studied a set of differently made electrical contacts. First type were standard $\mathrm{Ti} / \mathrm{Au}$ metal electrodes, the second type consisted of highly $n$-doped $\mathrm{ZnO}$ layer followed by a typical metal contact to $\mathrm{ZnO}(\mathrm{Ti} / \mathrm{Au})$. 


\section{Samples and experiments}

Substrates was GaN wafers grown by HVPE method with the electron concentration in the range of $10^{17} \mathrm{~cm}^{-3}$. Three types of samples was prepared:

1. with the standard $\mathrm{Ti} / \mathrm{Au}$ contacts (contacts annealed) - reference sample,

2. with the $50 \mathrm{~nm}$ high- $n$ type $\mathrm{ZnO}$ and the standard $\mathrm{Ti} / \mathrm{Au}$ contacts (contacts not annealed) - sample $50 \mathrm{ZnO}$,

3. with the $100 \mathrm{~nm}$ high- $n$ type $\mathrm{ZnO}$ and the standard $\mathrm{Ti} / \mathrm{Au}$ contacts (contacts annealed) — sample $100 \mathrm{ZnO}$

The highly- $n$ type $\mathrm{ZnO}$ layers were prepared by ALD at temperature of $200^{\circ} \mathrm{C}$ in Savannah-100 reactor from Cambridge NanoTech on both sides of wafers [2]. The diethylzinc (DEZn) and deionized water was used as zinc and oxygen precursors, respectively. Obtained layers were polycrystalline and conductive with the electron concentration on the order of $10^{20} \mathrm{~cm}^{-3}[3]$.

The $\mathrm{Ti} / \mathrm{Au}$ metallization was electron beam deposited on both sides of samples. Thickness of layers were $30 \mathrm{~nm}$ and $50 \mathrm{~nm}$, respectively. The metallization on the upper side of samples (N-side of GaN wafer) was square-shaped with dimensions of $300 \times 300 \mu \mathrm{m}^{2}$. Distance between metal pads was $50 \mu \mathrm{m}$. Bottom side of samples (Ga-side of $\mathrm{GaN}$ wafer) was fully covered by $\mathrm{Ti} / \mathrm{Au}$ contact.

The contacts on reference sample and $100 \mathrm{ZnO}$ sample was annealed at rapid thermal annealing (RTA) furnace during $1 \mathrm{~min}$ at the temperature of $300^{\circ} \mathrm{C}$ in nitrogen atmosphere [4]. The contacts on $50 \mathrm{ZnO}$ sample was prepared without annealing.

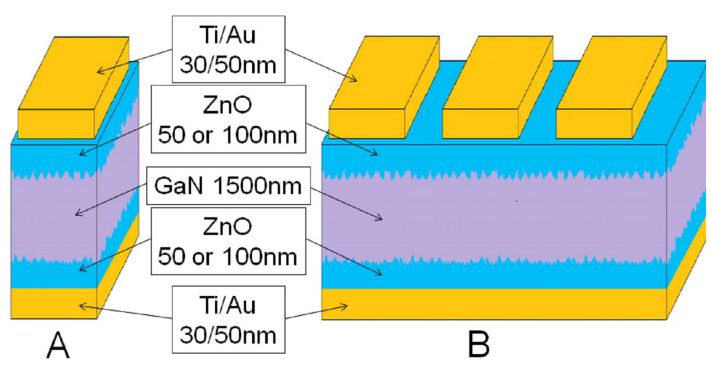

Fig. 1. Schematic view of samples: (A) for $I-V$ characteristic measurements, (B) for TLM measurements.

Two kinds of measurements on each sample were done (see Fig. 1):

1. classical current-voltage characteristics $(I-V$ characteristics) - in that measurement current was flown between upper and bottom contacts,

2. pseudo-transfer length method (TLM) measurements [5] — in those measurements current was flown between metal area on upper side and distance between contacts was changed. It is important to notice here that the square shaped metallization pads were different from the standard TLM pattern and could introduce a substantial error in the measurements, especially of the material resistivity.

Electrical measurements were performed with currentvoltage source Keithley 236 Source-Measure Unit. Scanning electron microscope (SEM) images were done by Hitachi tabletop microscope TM-1000.

\section{Experimental results}

The current-voltage $(I-V)$ characteristics from the first reference sample showed highly nonlinear behavior shown in Fig. 2. To determine the contact resistance and sample resistance the pseudo-TLM was applied. The geometry of the structure used for TLM measurement is shown in Fig. 1B. The inset to Fig. 2 demonstrates the dependence of the TLM resistance on the distance between the electrodes. We see the negative slope of the dependence indicating high resistance of the material. High non-linearity of the contacts does not allow for quantitative determining values of material and contact parameters.

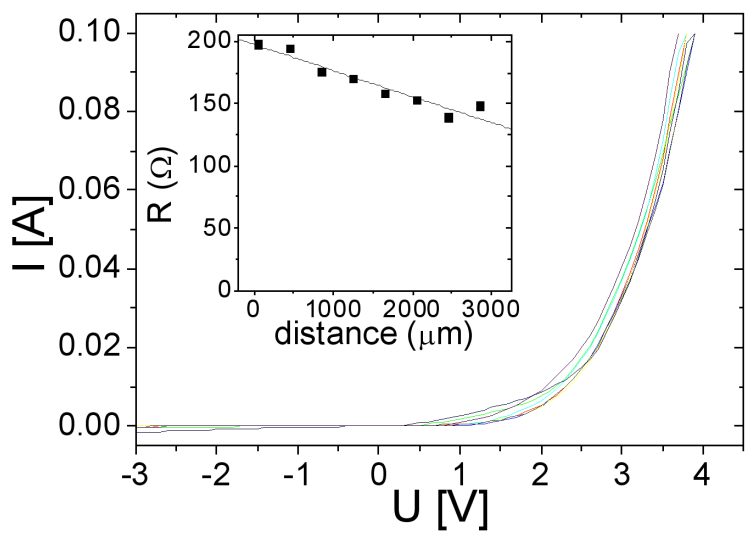

Fig. 2. $\quad I-V$ characteristics for sample without $n-\mathrm{ZnO}$ (reference): inset shows TLM plot with negative slope.

Figure 3 demonstrates the second type of contact the combination of $50 \mathrm{~nm}$ thick $\mathrm{ZnO}$ layer completed with $\mathrm{Ti} / \mathrm{Au}$ electrode. The contact was not annealed. We see the immediate improvement in the electrical characteristics. The contact though not yet completely linear allows for the observation of almost linear dependence of the TLM resistance on the electrode distance. From the inset of Fig. 3 we can obtain the value of contact resistivity as $19 \times 10^{-3} \Omega \mathrm{cm}^{2}$.

In order to further improve the contacts we increased $\mathrm{ZnO}$ layer thickness up to $100 \mathrm{~nm}$ (improvement in the layer continuity) and annealed the contact at $300{ }^{\circ} \mathrm{C}$ in nitrogen. Figure 4 shows the characteristics of such improved version of the contact layer. This time we can 


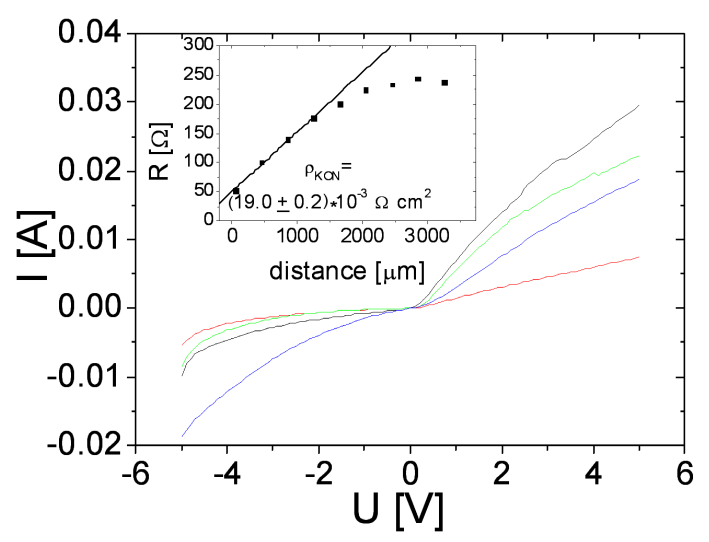

Fig. 3. $I-V$ characteristics for sample with $50 \mathrm{~nm}$ $n$-ZnO (contacts without annealing): inset shows TLM plot with nonlinear dependence of resistivity on distance.

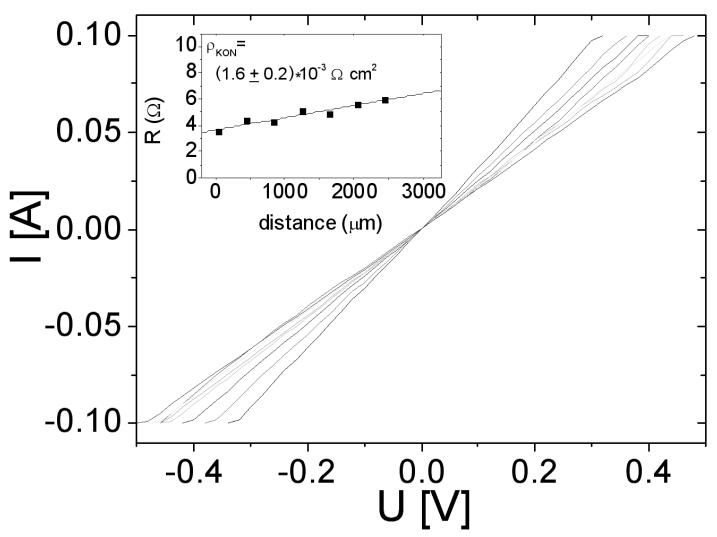

Fig. 4. $I-V$ characteristics for sample with $100 \mathrm{~nm}$ $n$-ZnO (with annealed contacts): inset shows TLM plot with linear dependence of resistivity on distance.

see very good contact linearity corresponding to the contact resistivity of $1.6 \times 10^{-3} \Omega \mathrm{cm}^{2}$. From the slope of the distance-resistance dependence we can calculate the GaN resistance which is of the order of $10^{-3} \Omega \mathrm{cm}$. Value of contact resistivity is sufficient for the most of the devices. For example for the typical laser diodes of dimensions of
$300 \times 700 \mu \mathrm{m}^{2}$, the bottom $n$-type contact would have the resistance of only $0.6 \Omega$.

Thus the presented here method may represent an interesting and useful alternative for the conventional metal contacts to $n$-type GaN of low-medium electron concentration.

\section{Conclusions}

The standard $\mathrm{Ti} / \mathrm{Au}$ metal contacts for bulk GaN with low electron concentration exhibit very poor properties (nonlinear character of $I-V$ curve and negative slope in TLM). The contacts without annealing for the same bulk GaN but with $50 \mathrm{~nm} \mathrm{ZnO}$ sublayer are better, but still nonlinear. The $\mathrm{Ti} / \mathrm{Au}$ annealed contacts for bulk GaN with $100 \mathrm{~nm}$ highly- $n$ type $\mathrm{ZnO}$ sublayer showed very good linearity and a low resistance of $1.6 \times 10^{-3} \Omega \mathrm{cm}^{2}$.

\section{Acknowledgments}

The research was partially supported by the European Union within European Regional Development Fund, through grant Innovative Economy (POIG.01.01.02-00$-008 / 08)$

\section{References}

[1] E. Guziewicz, M. Godlewski, T. Krajewski, Ł. Wachnicki, A. Szczepanik, K. Kopalko, A. Wójcik-Głodkowska, E. Przeździecka, W. Paszkowicz, E. Łusakowska, P. Kruszewski, N, Huby, G. Tallarida, S. Ferrari, J. Appl. Phys. 105, 122413 (2009).

[2] T. Krajewski, E. Guziewicz, M. Godlewski, L. Wachnicki, I.A. Kowalik, A. Wojcik-Glodkowska, M. Lukasiewicz, K. Kopalko, V. Osinniy, M. Guziewicz, Microelectron. J. 40, 293 (2009).

[3] N. Huby, S. Ferrari, E. Guziewicz, M. Godlewski, V. Osinniy, Appl. Phys. Lett. 92, 023502 (2008).

[4] Han-Ki Kim, Sang-Heon Han, Tae-Yeon Seong, Won-Kook Choi, Appl. Phys. Lett. 77, 1647 (2000).

[5] D.K. Schroder, Semiconductor Material and Device Characterization, 2nd ed., Wiley-Interscience, New York 1998. 